OPEN ACCESS

Edited by:

Francesca Lanfranconi, Victoria University, Australia

Reviewed by: Andrea Rigamonti, ASST Lecco, Italy Juergen Konczak,

University of Minnesota, United States

*Correspondence:

Ayman A. Mohamed amohamed@gelisim.edu

Specialty section: This article was submitted to Exercise Physiology, a section of the journal Frontiers in Physiology

Received: 24 November 2018 Accepted: 10 September 2019 Published: 01 October 2019

Citation:

Mohamed AA (2019) Can Proprioceptive Training Reduce Muscle Fatigue in Patients With Motor Neuron Diseases? A New Direction of Treatment. Front. Physiol. 10:1243. doi: 10.3389/fphys.2019.01243

\section{Can Proprioceptive Training Reduce Muscle Fatigue in Patients With Motor Neuron Diseases? A New Direction of Treatment}

\author{
Ayman A. Mohamed* \\ Department of Physiotherapy and Rehabilitation, School of Health Sciences, Istanbul Gelisim University, Istanbul, Turkey
}

Muscle fatigue is a serious problem in patients with motor neuron diseases (MNDs). It commonly disturbs both daily life activity and rehabilitation tolerance. A particular concern should be taken when MNDs occur in older ages. Older patients with MNDs usually have a worse clinical presentation and a lower survival rate. This could increase the occurrence of muscle fatigue. Muscle fatigue occurs due to a dysfunction in either motor or sensory systems. Current exercise interventions performed to decrease the occurrence of muscle fatigue focused only on treating motor causes of muscle fatigue. It has been demonstrated that these interventions have a high debate in their effectiveness on decreasing the occurrence of muscle fatigue. Also, these exercise interventions ignored training the affected sensory part of muscle fatigue, however, the important role of the sensory system in driving the motor system. Thus, this review aimed to develop a novel exercise intervention by using proprioceptive training as an intervention to decrease the occurrence of muscle fatigue in patients with MNDs particularly, older ones. The physiological effects of proprioceptive training to decrease the occurrence of muscle fatigue could include two effects. The first effect includes the ability of the proprioceptive training to increase the sensitivity of muscle spindles as an attempt to normalize the firing rate of $\alpha$-motoneurons, which their abnormalities have major roles in the occurrence of muscle fatigue. The second effect includes its ability to correct the abnormal movement-compensations, which develop due to the biomechanical constraints imposed on patients with MNDs.

Keywords: motor neuron diseases, muscle fatigue, older patients, proprioceptive, training

\section{INTRODUCTION}

Muscle fatigue is a common problem in patients with motor neuron diseases (MNDs) (Gibbons et al., 2013). MNDs are defined as a group of diseases in which there is a progressive degeneration of motor neurons (Quansah and Karikari, 2015). MNDs include three main subtypes. The first subtype includes disorders which affect lower motor neurons, such as spinal muscular atrophy (SMA) and spinobulbar muscular atrophy (SBMA or Kennedy's disease). The second subtype includes disorders which affect upper motor neurons, such as spastic paraplegias and primary 
lateral sclerosis (PLS). The last subtype includes disorders which affect both upper and lower motor neurons, such as amyotrophic lateral sclerosis (ALS) (Figlewicz and Orrell, 2003).

One of the common complaints in patients with MNDs is muscle fatigue (McElhiney et al., 2009; Abraham and Drory, 2012; Gibbons et al., 2018). Muscle fatigue is defined as the timerelated reduction in the maximum force-production capacity of the muscle. Muscle fatigue is one of the most common complaints occur in patients with MNDs. McElhiney et al. (2009) have demonstrated that muscle fatigue affects approximately $44 \%$ of patients with MNDs. It has been reported that there are several causes for the occurrence of muscle fatigue in MNDs. Most studies have demonstrated that neurodegenerative cause is the main cause of muscle fatigue in patients with MNDs, while the neuromuscular transmission, and muscle metabolism are normal in those patients (Abraham and Drory, 2012). In contrast, other studies have stated that patients with ALS usually have a neuromuscular junction disassembly and muscle denervation and this abnormality of the neuromuscular junction is trademark feature of ALS onset and progression. Other studies have shown that there is a severe abnormality in the mitochondrial function in patients with ALS (Bowling et al., 1993; Dupuis et al., 2005; Vandoorne et al., 2018).

However, MNDs are common in adults, this previous debate increases in older ages because older patients with MNDs usually experience more complex clinical presentation and lower survival rate (Eisen et al., 1993; Forbes et al., 2004; Broussalis et al., 2018). It has been demonstrated that the prognosis is much worse in older patients with MNDs than younger ones (Tysnes et al., 1994). Older patients with MNDs usually have a higher incidence of bulbar symptoms than younger ones and this bulbar onset presents in at least half of all patients with MNDs over 80 years (Eisen et al., 1993; Forbes et al., 2004; Broussalis et al., 2018). Also, older patients with MNDs could experience more occurrence of muscle fatigue during exercises than younger ones because aging produces an abnormality in normal function and firing rate of the $\alpha$-motoneurons (Soderberg et al., 1991; Knight and Kamen, 2007; Watanabe et al., 2016), which is considered one of the main causes of muscle fatigue.

Furthermore, it has been shown that elderlies usually experience a high degree of muscle fatigue during exercise rather than younger ones (Klass et al., 2007; Mcneil and Rice, 2007; Kent-Braun, 2009). Klass et al. (2007) investigated the effect of aging on the fatigability of ankle dorsiflexor muscles throughout concentric and eccentric contractions. They found that fatigability increased progressively with aging and they argued this increase in fatigability to peripheral alterations occurred in $\mathrm{Ca}^{2+}$-controlled excitation-contraction coupling process and neuromuscular propagation. Thus, the occurrence of MNDs in older ages could cause further alteration in the $\mathrm{Ca}^{2+}$-controlled excitation-contraction coupling process and neuromuscular propagation.

Moreover, it has been demonstrated in the literature that there is a change in muscle fibers with both aging and MNDs. With aging, type I muscle fibers usually transform to type II, which increases the occurrence of muscle fatigue during exercises (Mcneil and Rice, 2007; Kent-Braun, 2009). Also, it has been shown that with MNDs, there are atrophic changes in muscle fibers with mild denervation. Thus, older patients with MNDs could experience more changes in muscle fibers than younger ones due to the combination of aging and MNDs effects. Thus, older patients with MNDs should not be neglected from future revisions.

Current exercise interventions performed to treat muscle fatigue in patients with MNDs are few and their qualities are very low (Gibbons et al., 2018). Thus, it is impossible to reach strong conclusions about the effectiveness of these interventions to reduce the occurrence of muscle fatigue in patients with ALS/MND (Gibbons et al., 2018). Generally, exercise interventions performed to treat muscle fatigue have assumed that muscle fatigue occurs due to dysfunction in motor control. This dysfunction occurs due to failure in one or more mechanisms included in the voluntary muscle contraction. This failure can occur in any area along the neuromuscular system, including the motor cortex, signals from the motor cortex to motoneurons, signals from motoneurons to muscle, neuromuscular junction coupling in muscle, or actin-myosin links (Light et al., 2010).

Also, current exercise interventions included either grading exercise intensity (Wallman et al., 2004), increasing rest period (Nogueira et al., 2012), using mild training intensity (Dennett et al., 2016), or using massage for the fatigued muscle (Nunes et al., 2016). The effectiveness of these exercise interventions is still in debate. Some studies (Morriss et al., 1996; Friedberg, 2002; Wallman et al., 2004) have demonstrated that increasing physical activity or grading exercise intensity is beneficial in decreasing the occurrence of muscle fatigue. In contrast, other studies (Black et al., 2005; Oh et al., 2016) have shown that increasing physical activity or grading exercise intensity has no effect on reducing the occurrence of muscle fatigue.

It has been shown in the literature that muscle fatigue does not occur due to dysfunction in the motor control only, however, it occurs due to dysfunction in both motor and sensory systems (Light et al., 2010). Several studies have shown that MNDs affect sensory neurons besides motor neurons (Anagnostou et al., 2005; Pugdahl et al., 2007; Vaughan et al., 2015). Pugdahl et al. (2007) conducted a study to detect the presence of any dysfunction in sensory neurons in patients with ALS. They found that about $22.7 \%$ of the included patients had an abnormality in the conduction time of at least one sensory nerve. Anagnostou et al. (2005) conducted a study to detect the presence of any dysfunction in sensory neurons in children with SMA. They found that children with SMA had dysfunctions in the conduction time of sensory nerves. Recently, Vaughan et al. (2015) conducted a study to detect the presence of any dysfunction of the proprioceptive system in mice with ALS. They found that these mice had significant degenerations in nerve endings of the proprioceptive system.

Also, it has been demonstrated in several studies that muscle fatigue has sensory receptors responsible for sensing and developing muscle fatigue (Light et al., 2010; Boyas and Guével, 2011; Staud, 2012; Nunes et al., 2016; Kuppuswamy, 2017). However, the vital role of the sensory system in driving motor control (Riemann and Lephart, 2002), till now 
there is no exercise intervention focused on improving the function of the sensory element of muscle fatigue. It has been shown in the literature that the sensory mechanism of muscle fatigue starts from mechanoreceptors and metaboreceptors. These receptors are responsible for generating the sensation of muscle fatigue (St Clair Gibson et al., 2003; Light et al., 2010). Mechanoreceptors are primary receptors of muscle fatigue and they are sensitive to changes in muscle strain (St Clair Gibson et al., 2003; Light et al., 2010). While metaboreceptors are secondary receptors of muscle fatigue and they are sensitive to changes in the number of metabolites created by muscle contraction (St Clair Gibson et al., 2003; Light et al., 2010).

Mechanoreceptors are also the same receptors of the proprioception. The sensitivity and function of mechanoreceptors can be improved both neurologically or morphologically by performing proprioceptive training (Kaya, 2016). Thus, it is reasonable to suppose that performing a proprioceptive training to the fatigued muscle could improve the function of mechanoreceptors within this muscle. Consequently, this could be an effective modality to reduce the occurrence of muscle fatigue in patients with MNDs, particularly older patients who have a worse clinical presentation, and a low survival rate. Thus, this review aimed to demonstrate possible physiological mechanisms of proprioceptive training as an exercise intervention to treat muscle fatigue in patients with MNDs, particularly older ones.

This review included seven subtopics, the previous mechanisms of muscle fatigue in MNDs, the proprioception dysfunctions in MNDs, the mechanism of the sensation of muscle fatigue, the possible physiological effects of proprioceptive training on decreasing muscle fatigue in MNDs, the effects of proprioceptive training to correct the imposed biomechanical constraints in MNDs, the effects of aging on pathological degeneration of motor and sensory neurons in patients with MNDs, and the physiological effects of proprioceptive training to create theoretical bases to fight the MNDs in elderlies.

\section{The Previous Mechanisms of Muscle Fatigue in MNDs}

It has been reported that muscle fatigue in MNDs occurs as a result of a defect in lower motor neurons. This defect causes a failure of motor units to provide the needed levels of activity, consequently, peripheral muscle fatigue occurs (Abraham and Drory, 2012). In the literature, neurodegenerative causes are the main causes of muscle fatigue. These neurodegenerative causes include any dysfunction of microglia, glutamate excitotoxicity, misfolded proteins, mitochondrial dysfunction, or oxidative stress (Abraham and Drory, 2012).

Vucic et al. (2007) investigated the alteration of axonal excitability occurred after an induced voluntary contraction to recognize peripheral mechanisms of muscle fatigue in patients with ALS. They found that patients with ALS had a membrane hyperpolarization. This membrane hyperpolarization caused an increase in the threshold occurred after the voluntary contraction in patients with ALS compared with controls. They argued this membrane hyperpolarization to the abnormality in either the $\mathrm{Na}^{+} / \mathrm{K}^{+}$pump or firing rate of motor neurons. They also found that there was a dysfunction in the $\mathrm{Na}+/ \mathrm{K}+$ ATPase, which might cause a loss of motor neurons.

Sharma et al. (1995) examined possible mechanisms of muscle fatigue in patients with ALS. They measured muscle force, energy metabolism, and muscle activation pattern. They used the phosphorus-3 1 magnetic resonance spectroscopy to measure muscle force and energy metabolism, and the neurophysiological measures and magnetic resonance imaging to measure the muscle activation pattern. These measures were collected through a 25 min intermittent isometric contraction of the tibialis anterior muscle. They found that both tetanic and maximum voluntary force decayed in those patients more than controls. Also, they found that muscular activation impaired due to small proton signal intensities and amounts of energy metabolites. Lastly, they found that the neuromuscular transmission was nearly normal because amplitudes of the evoked compound of the muscle action potential were steady throughout the contraction.

However, the common belief that the neuromuscular transmission and muscle metabolism are normal in patients with MNDs, several studies have demonstrated that patients with MNDs experience abnormalities in the neuromuscular transmission, and mitochondrial function (Bowling et al., 1993; Dupuis et al., 2005; Rocha et al., 2013; Cappello and Francolini, 2017; Vandoorne et al., 2018). Cappello and Francolini (2017) have stated that patients with ALS usually have a neuromuscular junction disassembly and muscle denervation. Additionally, Rocha et al. (2013) have demonstrated that the degeneration of the neuromuscular junction is a trademark feature of ALS onset and progression. Also, It has been shown in several studies that there is a severe abnormality in the mitochondrial function in patients with ALS (Bowling et al., 1993; Dupuis et al., 2005; Vandoorne et al., 2018). Thus, this abnormality in the neuromuscular transmission and mitochondrial function should be considered as a source of muscle fatigue in patients with MNDs in the future.

\section{The Proprioception Dysfunctions in MNDs}

Motor neuron diseases significantly disturb the whole proprioceptive system. Several studies have demonstrated that MNDs usually disturb a variety of cells, such as Renshaw and Glial cells in the spinal cord (Haidet-Phillips et al., 2011; Mochizuki et al., 2011; Philips and Rothstein, 2014; Vaughan et al., 2015). Some studies used neurophysiological and neuroimaging analyses to detect the presence of any abnormality in the sensory neurons in patients with ALS. They found that about $20-60 \%$ of sensory neurons showed an abnormality in those patients (Hammad et al., 2007; Pugdahl et al., 2007). Other studies used a histological analysis to detect any abnormality in the sensory neurons. Also, they found that there was a significant degree of degeneration in these neurons and their axons (Dyck et al., 1975; Hammad et al., 2007).

One of the major sensory systems which has a vital role in driving motor control is the proprioceptive system 
(Vaughan et al., 2015). The degeneration of proprioceptive neurons, mainly Ia/II proprioceptors, would possibly have a significant effect on increasing the deterioration of $\alpha$-motoneurons. It has been demonstrated that proprioceptive (sensory) and $\alpha$-motor neurons are structurally and functionally connected. Proprioceptive information detected by mechanoreceptors delivers to $\alpha$-motoneurons via monosynaptic connections to adjust their actions. Thus, any loss of the proprioceptive mechanism could highly affect $\alpha$-motoneurons function (Vaughan et al., 2015).

Two animal studies have demonstrated that MNDs significantly affect the proprioceptive mechanism. Mentis et al. (2011) conducted a study to detect the presence of any early signs of malfunction in the sensory-motor connectivity in mice with SMA. They found that mice with SMA experienced a decrease in proprioceptive reflexes and a decrease in function and number of proximal dendrites and motor neuron synapses. These abnormalities raised early through the disease and they accompanied the affection of motor neurons. Also, Vaughan et al. (2015) conducted a study to detect the presence of any degrees of degeneration in nerve endings of the proprioceptive system in mice with ALS. They found that peripheral nerve endings of the proprioceptive system experienced a significant degree of degeneration, particularly types Ia/II. This degeneration occurred early prior to the presence of any neurological symptoms or loss of any central projecting nerve branches.

To the best of our knowledge, there was no human study in the literature demonstrated the effect of MNDs on the proprioceptive system. Only a human study conducted by Hammad et al. (2007) to detect the presence of any degree of sensory involvement in patients with ALS. They found that approximately $32 \%$ of those patients had affection in the sensory system, about $27 \%$ of those patients had abnormalities in the amplitudes of nerve action potentials of the sural sensory nerve, and $91 \%$ of those patients had pathological anomalies in the sensory system. Also, they found that large-caliber myelinated fibers got the most affection (73\%), while small-caliber myelinated fibers got the least affection (23\%). Furthermore, they found that there was a significant degree of degeneration in both axons and myelin sheaths.

\section{The Mechanism of the Sensation of Muscle Fatigue}

The sensation of muscle fatigue is a complex phenomenon. It occurs on both conscious and unconscious perceptions. The sensation of fatigue occurs in the same way by which the body senses any change in its functions, such as the decrease in the heart rate occurs as a consequence to any increase in cardiac output, feeling of an elevated muscle activity rate occurs as a consequence to any elevation in the power generation, which occurs in response to a rise in its physical activity levels, the breathlessness occurs in response to any increase in the ventilation, and the sensation of warm and gummy occurs with any increase in the temperature or sweating (St Clair Gibson et al., 2003).

The sensation of muscle fatigue starts with a change in a particular component during the physical activity, such as a change in sensation of strain in working muscles and/or joints (St Clair Gibson et al., 2003), accumulation of muscle metabolites (Fitts, 1994; Green, 1997), or depletion of substrates (Balsom et al., 1999; McConell et al., 1999). These peripheral changes are sensed by either mechanoreceptors (Pandolf et al., 1975; Mihevic, 1981) or metaboreceptors (Rotto and Kaufman, 1988; Bongiovanni and Hagbarth, 1990). Then, this sensory data reaches the brain to inform it by the level of exertion or fatigue in working muscles (St Clair Gibson et al., 2003).

Mihevic (1981) has demonstrated that the perception of exertion relies on input data from both "muscle and cardiorespiratory system." This data includes a feedback data about changes in the muscle strain (the primary source for the sensation of fatigue) and a feedback data from the cardiorespiratory system about the depletion in the number of metabolites or substrates (the secondary source of the sensation of fatigue). This study came in accordance with the study of Stamford and Noble (1974), who found that the proprioceptive feedback, precisely from the Golgi tendon organ, was the primary mechanism of the perception of exertion.

Hutton and Nelson (Nelson and Hutton, 1985; Hutton and Nelson, 1986) also conducted two studies to investigate the activity of mechanoreceptors in the fatigued gastrocnemius muscle during ramp stretch in cats. The first study (Mochizuki et al., 2011) investigated the sensitivity of Golgi tendon organs in fatigued gastrocnemius muscle. They found that with ramp stretch, there was a significant decrease in response latencies of Ib nerve types. This decrease presented regardless of any rise in twitch tension or change in peak and static tension. Also, White and Hall (2018) reached the same results and they added that during muscle fatigue the Golgi tendon organs had a tendency to preserve a fixed level of force which could be the cause of the continuous reduction in muscle force.

The second study (Nelson and Hutton, 1985) investigated the sensitivity of muscle spindles in the fatigued gastrocnemius muscle. They found that during static stretching of the fatigued muscle, there was a decline in response latency to any displacement, a rise in the mean frequency, and an increase in resting discharge. While at rest, the frequency of firing to vibration significantly increased in both Ia and IIa nerve fiber types. Also, they found that the sensitivity of cats to different positions significantly decreased with the occurrence of muscle fatigue.

The association between proprioceptive dysfunction and muscle fatigue has been demonstrated in the literature (Ribeiro et al., 2007; Gear, 2011). Ribeiro et al. (2007) investigated the effect of induced muscle fatigue on the knee position sense in elderlies. They performed 30 successive maximal gravity adjusted concentric contractions to knee flexors and extensors using an isokinetic dynamometer. They found that with muscle fatigue, the absolute angular error significantly increased, and the peak torque of knee muscles significantly declined. Gear (2011) investigated the effect of various levels of muscle fatigue of the hamstring muscle on the position sense of the knee joint. In this study, an isokinetic exercise through an angular range of motion used to produce muscle fatigue. Three levels of muscle fatigues were examined, including 90\% (mild fatigue), 70\% (moderate 
fatigue), and 50\% (maximum fatigue) of hamstring peak torque. $\mathrm{He}$ found that there was a significant decrease in the position sense of the knee joint at $90 \%$ and $50 \%$ of muscle fatigue.

\section{The Possible Physiological Effects of Proprioceptive Training on Muscle Fatigue in MNDs}

It has been reported in the literature that the proprioceptive system has three functions (Jha et al., 2017). It protects joints from excessive and injurious movements via a reflexive mechanism in response to proprioception afferent feedback. It assists in the stabilization of joints during a static posture. Finally, it promotes a better performance of complex movements in more precise coordinated manners. Several studies have stated that proprioceptive training can achieve significant effects on improving motor control dysfunctions in almost musculoskeletal or neurological disorders. These improvements occurred in balance control (Tibone et al., 2013), pain level (McCaskey et al., 2014), motor learning (Aman et al., 2014), and walking parameters (Yong and Lee, 2017).

However, the high value of proprioceptive training in the field of rehabilitation, its effect on decreasing the occurrence of muscle fatigue in patients with MNDs has not been demonstrated yet. The main effect of proprioceptive training on reducing the occurrence of muscle fatigue includes its ability to normalize the firing rate of motor neurons. It has been demonstrated that the decrease or stoppage of the firing of motor neurons has a significant role in reducing the muscular force and developing muscle fatigue (Wan et al., 2017). One of the major causes of the decrease in muscle spindle activity is the decrease in the motor neuron firing rate, which reduces the firing rate of group Ia muscle afferents. Thus, an increase in the presynaptic inhibition and decreasing the firing rate of the motor neurons occur (Brerro-Saby et al., 2008; Vie et al., 2013). Improving the muscle spindle activity by proprioceptive training could help in renormalizing the firing rate of group Ia muscle afferents, presynaptic inhibition and firing rate of motor neurons (Ribot-Ciscar et al., 2000; Hospod et al., 2007; De Luca and Kline, 2012).

Hospod et al. (2007) examined the effect of proprioceptive training on the muscle spindle activity arising from the common peroneal nerve. They found that Ia afferent responses changed significantly after the performance of proprioceptive training. The change in Ia afferent included an increase in the variability of discharge, a decrease in depth of modulation, and a change in spontaneous activity. Potvin and Fuglevand (2017) developed a phenomenological model of motor unit fatigue as a controllable resource to expect muscle fatigue for several tasks and to demonstrate different contractile responses of motor units. This phenomenological model demonstrated that normalization of the firing rate of motor neurons caused an increase in muscle performance and a decrease in the occurrence of muscle fatigue.

Normalization of the firing rate of motor neurons consequently could help in normalizing the amount of calcium released from calcium channels in the sarcoplasmic reticulum and skeletal muscles. The normal release of calcium helps in decreasing the incidence of muscle fatigue because the depletion of calcium is considered one of the main causes of muscle fatigue (Fryer et al., 1995). Muscle spindles activate intrafusal muscle fibers through the activation of gamma motoneurons, which increases strain on the sensory region. Then, through a reflex action intermediated by muscle spindle afferents, an increase in $\alpha$-motoneurons activity, stimulation of the extrafusal muscle fibers, and occurrence of muscle contraction occur afterward (Edman et al., 2002).

Kuo and Ehrlich (2015) have demonstrated that the contraction of the extrafusal muscle fibers occurs due to the activation of $\alpha$-motoneurons which stimulates the release of the acetylcholine at the neuromuscular junction. The released acetylcholine spreads across the synaptic cleft and activates nicotinic acetylcholine receptors over the motor endplate. The activation of nicotinic acetylcholine receptors causes an influx of cations (sodium and calcium) then the depolarization of the muscle cell membrane occurs afterward. This depolarization triggers high numbers of voltage-gated sodium channels over the muscle membrane and causes initiation of the action potential.

The action potential spreads along the surface membrane and transverse tubules. In transverse tubules, the action potential is sensed by the dihydropyridine receptors (voltage-sensor molecules). This mechanism sequentially opens the calcium release channels in the sarcoplasmic reticulum and skeletal muscles. These channels release calcium into the sarcoplasm (Fichna et al., 2015). Then, calcium binds with the troponin to move the tropomyosin far away of the myosin-binding area on actin. This initiates the cross-bridge cycling and muscle contraction (MacIntosh et al., 2012). After muscle contraction, the calcium is removed from the cytoplasm by Ca2+ATPase enzyme. This causes a return of tropomyosin to its blocked location and the relaxation to occur (MacIntosh et al., 2012). Using proprioceptive training could help in the normalization of calcium release mechanism by increasing the muscle spindle activity; this could assist significantly in reducing the incidence of muscle fatigue. The pathological mechanisms responsible for the occurrence of muscle fatigue in patients with MNDs and the effect of proprioceptive training on the renormalization of these mechanisms are illustrated in Figure 1.

\section{The Effects of Proprioceptive Training to Correct the Imposed Biomechanical Constraints in MNDs}

One of the common signs of MNDs is muscle weakness. The pattern of weakness can be either distal to proximal (upper motor neuron disease) or proximal to distal (lower motor neuron disease) (Statland et al., 2015). This weakness occurs due to the degeneration of motor units of certain muscles according to the pattern of weakness for each type. Assuming that force requirements are the same to keep the body erect or produce any movement, thus the load increases in a pattern opposite to the weakness pattern of each type. Thus, movement compensations develop and a further increase in muscle fatigue occurs with any small load or activity. 


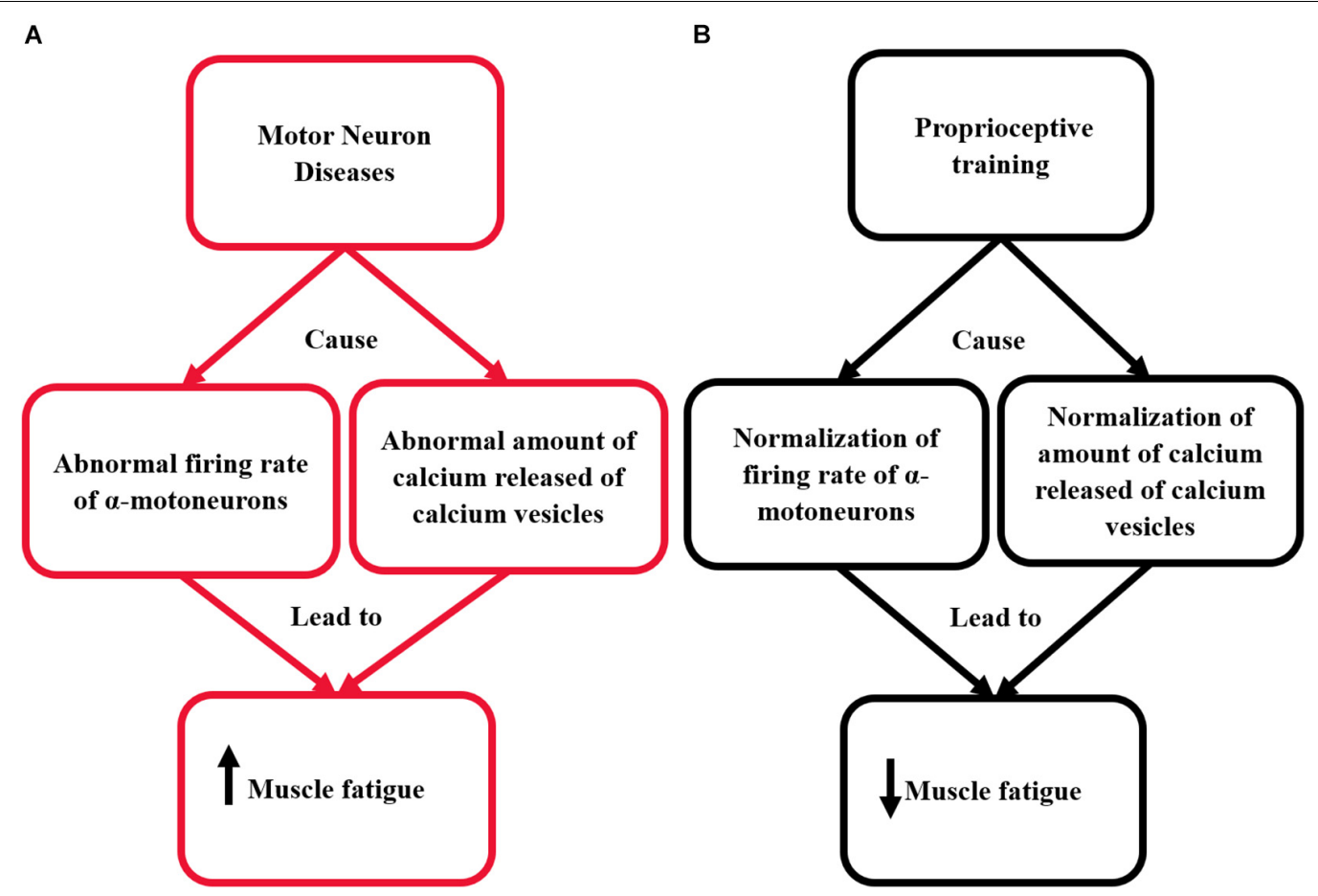

FIGURE 1 | This diagram illustrates the effect of proprioceptive training on re-normalizing the pathological mechanisms responsible for the occurrence of muscle fatigue in patients with MNDs. (A) The pathological mechanism responsible for the occurrence of muscle fatigue in patients with MNDs. (B) The effect of proprioceptive training on renormalizing the pathological mechanisms responsible for the occurrence of muscle fatigue in patients with MNDs.

Several studies (Wu and Ng, 2010; Wu and Shi, 2011; Radovanović et al., 2014; Witiuk et al., 2014; Grunseich and Fischbeck, 2015; Hausdorff et al., 2019) have demonstrated that patients with MNDs have functional movement-compensations. Grunseich and Fischbeck (2015) have demonstrated that patients with SMA usually have progressive leg weakness. This weakness is symmetrical and causes more muscle fatigue in order to keep the balance on uneven surfaces. Radovanovic et al. (2014) have demonstrated that patients with ALS have a longer gait cycle and smaller stride length compared to controls. Hausdorff et al. (2019) have shown also that patients with ALS have a longer cycle time and more decrease in cadence, stride length, and velocity compared to controls. They also have shown that patients with ALS spend less time on one leg (swing time) and more time on two legs (double support time).

Functional movement-compensations present in patients with MNDs can be improved by proprioceptive training. Proprioceptive training can adjust the motor control and correct these functional movement-compensations through both increasing patient awareness about the normal movements and correcting the abnormal ones (modulating motor control).

Proprioceptive training can modulate motor control through either central or peripheral mechanisms (Kaya, 2016). Centrally, Eriksson (2001) and Kaya (2016), have shown that proprioceptive training modifies proprioceptive input by modulating muscle spindle control and inducing plastic adjustments in the central nervous system (Vallbo and Al-Falahe, 1990). Peripherally,
Hutton and Atwater (1992) have shown that proprioception training causes morphological adaptations in the muscle spindles themselves. These morphological adaptations occur due to micro-adaptations occur to the intrafusal muscle fibers due to some metabolic alterations. Also, these macro-adaptations can occur due to a decline in the response latency of the stretch reflex and a rise in its amplitude.

A study conducted by myself and others (Mohamed et al., 2019) to correct the shrug sign which is a type of movement compensations usually develops in patients with adhesive capsulitis. However, adhesive capsulitis is a self-limiting disorder, this sign can prevent its full recovery. In our study, we developed a new proprioceptive training to correct the shrug sign. We found that this proprioceptive training decreased the shrug sign and helped in gaining more shoulder and scapular range of motion. The effects of proprioceptive training on decreasing movementcompensations responsible for increasing the occurrence of muscle fatigue in patients with MNDs are illustrated in Figure 2.

\section{The Effects of Aging on Pathological Degeneration of Motor and Sensory Neurons Occur in Patients With MNDs}

Several studies have shown that the incidence of MNDs among elderlies increases and those patients experience a worse clinical presentation and low survival rate than younger adults (Eisen et al., 1993; Norris et al., 1993; Forbes et al., 2004; Terao et al., 2006; Broussalis et al., 2018). Broussalis et al. (2018) 


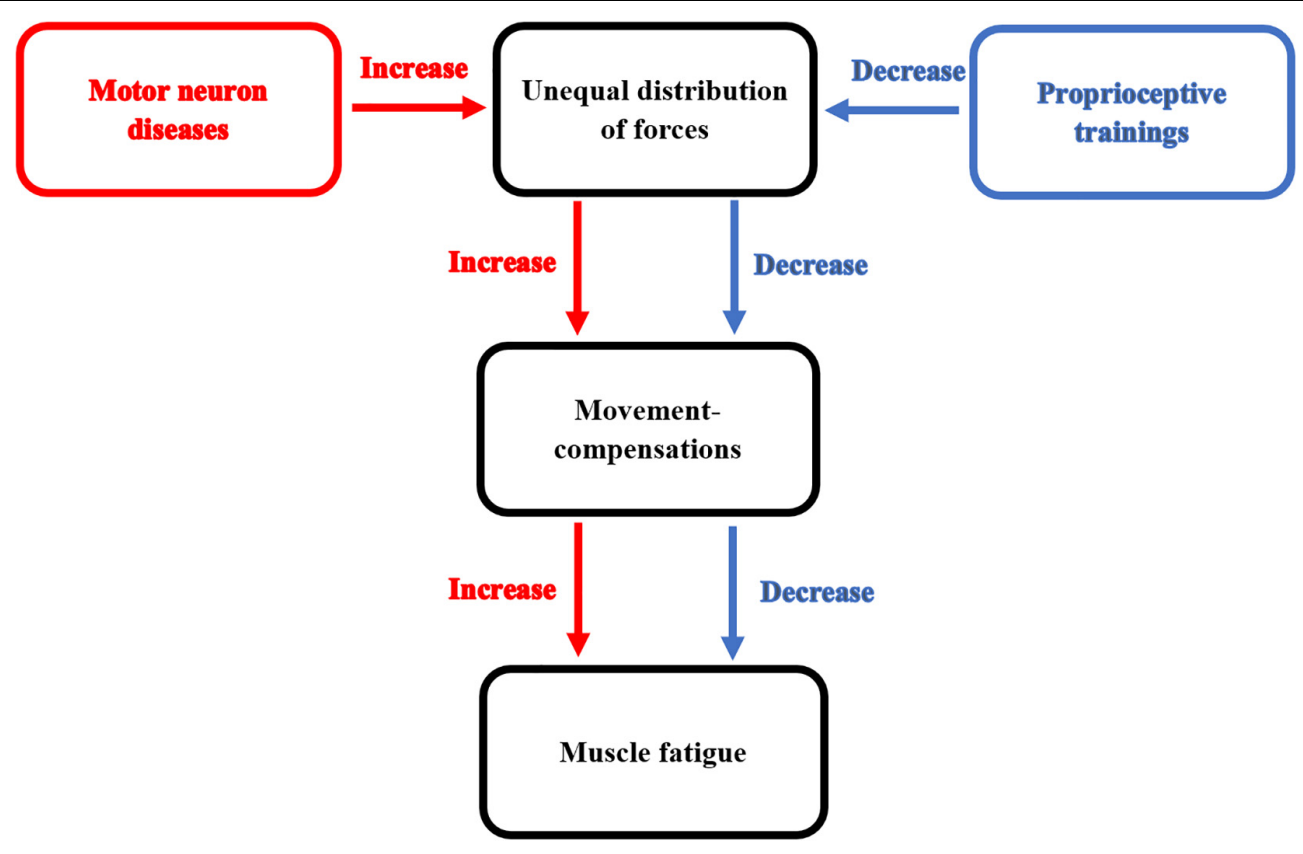

FIGURE 2 | This diagram illustrates the effect of proprioceptive training on decreasing movement-compensations responsible for increasing the occurrence of muscle fatigue in patients with MNDs.

conducted a study to investigate the onset of ALS among elderlies. They found that the majority of admitted patients were elderlies (age of older than 70). Forbes et al. (2004) conducted another study to investigate the clinical presentation of ALS in elderlies over 80 years. They found that clinical presentation and survival rate in elderlies with ALS were worse than younger adults. These results correspond with the study of Terao et al. (2006), who conducted a study to investigate the clinical presentation, and survival rate of ALS among older Japanese patients. They found older patients with ALS had worse survival rates and more complications than younger adults.

Aging is considered one of the key risk factors for the development of MNDs (Kurtzke, 1991; Hospital et al., 1993). Causes of the worse survival rate among older patients with MNDs are not clear yet. These causes might be mainly due to the deteriorating effects of aging on musculoskeletal and neurological systems (Kurtzke, 1991; Hospital et al., 1993). Aging usually causes atrophic changes in extrafusal muscle fibers (McKenzie et al., 2002; Hepple, 2003; Marzetti et al., 2012), degenerations of neuromuscular junctions (Valdez et al., 2010; Tintignac et al., 2015), physiological and cellular modifications in motor axons (Apel et al., 2009; Kang and Lichtman, 2013), and changes in the expression of genes that could critically change normal functions of neuromuscular junctions and skeletal muscles (McKenzie et al., 2002; Weisleder et al., 2006; Jang et al., 2011). Aging causes a decline in both the peripheral and central nervous system processing of sensory information (Nusbaum, 1999). Thus, these mechanisms could significantly cause more complication and worse survival rate among older patients with MNDs.

Furthermore, several studies (Herndon et al., 2002; Pan et al., 2011; Sann et al., 2012; Toth et al., 2012; Li et al., 2016) have examined the effect of age on neuronal tissues using animal models. Understanding these animal models can offer a vision into the bases of selective neuronal susceptibility in neurodegenerative disorders in humans. These animal studies have demonstrated that aging causes abnormal changes in neural axons within the spinal cord. These changes include swelling, waviness, defasciculation, and shrinkage of their diameter (Herndon et al., 2002). Also, aging causes abnormal changes in neurons. These changes include soma distortion, development of abnormal branches, and novel neurite-like projections from the soma (Pan et al., 2011; Tank et al., 2011; Toth et al., 2012). Furthermore, aging causes extensive structural changes in mechanosensory neurons and their microtubule networks (Pan et al., 2011; Toth et al., 2012). These structural changes can disorganize with distorted somas (Pan et al., 2011).

Other types of neurons also exhibit age-related morphological changes, such as dopaminergic neurons, chemosensory neurons, interneurons, and motor neurons. Aging causes morphological changes in the soma of the dopaminergic neuron (McCaskey et al., 2014), axon edging of GABAergic neurons, defasciculation of cholinergic axons in the anterior nerve cord (Pan et al., 2011), and ectopic branches from GABAergic axons (Tank et al., 2011). Aging causes a synaptic decline in the aged neurons, this occurs due to the decrease in the number of synaptic vesicles and size of presynaptic concentrations in the spinal cord (McCaskey et al., 2014).

Aging causes proteins such as SNB-1/synaptobrevin and RAB3 GTPase, to ectopically collect in synaptic axonal regions and dendrites (Li et al., 2016). Endosomal membrane compartments in aged GABAergic motor neurons disorganize too. 
These GABAergic motor neurons are important for constructing and reprocessing of synaptic vesicles (Sann et al., 2012). With aging, the presynaptic release of substances decreases in motor neurons and gradually deteriorates afterward, these substances are important for muscle contraction (Liu et al., 2013). Aging causes deterioration of the synaptic organization in the form of a decrease in the number of dendritic spines and the axonal transport, which is vital for synaptic maintenance (Chen and Hillman, 1999; Valdez et al., 2010). With aging, there is a malfunction in neuronal transporters released from synaptic vesicles. The malfunction of these transporters increases the speed of the synaptic decline and motor circuit malfunction. This might explain the chief role of axonal transport in the preservation of synaptic structural integrity through human life (Li et al., 2016).

\section{The Physiological Effects of Proprioceptive Training to Create Theoretical Bases to Fight the MNDs in Elderlies}

However, MNDs significantly affect human life quality and mobility (Simmons, 2013), abnormal structural, and morphological changes occur with aging could aggravate these adverse effects, and speed the degeneration rate occurs to $\alpha$-motoneurons in patients with MNDs. Normalization of $\alpha$-motoneurons using proprioceptive training might be a good intervention to fight the occurrence of MNDs in older ages. This can be accomplished by increasing the sensitivity of mechanoreceptors particularly, muscle spindles and Golgi tendon organs which significantly decrease in older ages (Kararizou et al., 2005; Liu et al., 2005; Ribeiro and Oliveira, 2007). It has been demonstrated that increasing the sensitivity of mechanoreceptors could normalize the firing rate of $\alpha$-motoneurons. This could decrease the disruption of the function of $\alpha$-motoneurons, the calcium release, and the ATPase enzyme; these mechanisms mainly present with MNDs (Sharma et al., 1995; Ellis et al., 2003; Masson et al., 2014; Nijssen et al., 2017). Thus, using proprioceptive training could be a useful tool to slow down the deterioration in the function of $\alpha$-motoneurons.

The effectiveness of proprioceptive training on modulating the abnormal tone and improving manual control has been shown with other neuro-degenerative disorders (Shumaker, 1980;

\section{REFERENCES}

Abraham, A., and Drory, V. E. (2012). Fatigue in motor neuron diseases. Neuromuscul. Disord. 22(Suppl. 3), S198-S202. doi: 10.1016/j.nmd.2012.10.013

Aman, J. E., Elangovan, N., Yeh, I.-L., and Konczak, J. (2014). The effectiveness of proprioceptive training for improving motor function: a systematic review. Front. Hum. Neurosci. 8:1075. doi: 10.3389/fnhum.2014.01075

Anagnostou, E., Miller, S. P., Guiot, M.-C., Karpati, G., Simard, L., Dilenge, M. E., et al. (2005). Type I spinal muscular atrophy can mimic sensory-motor axonal neuropathy. J. Child Neurol. 20, 147-150. doi: 10.1177/08830738050200022101

Apel, P. J., Alton, T., Northam, C., Ma, J., Callahan, M., Sonntag, W. E., et al. (2009). How age impairs the response of the neuromuscular junction to nerve transection and repair: an experimental study in rats. J. Orthop. Res. 27, 385-393. doi: 10.1002/jor.20773
Bieñkiewicz et al., 2013; Shih et al., 2016; Wang et al., 2018). Bieñkiewicz et al. (2013) conducted a study to investigate the effect of proprioceptive training by using visual biofeedback on bradykinetic movements of the hand in patients with Parkinson's disease. They found that temporal features of hand movements significantly moderated by using visual biofeedback. These improvements included an improvement of muscle tone, movement time, and peak velocity.

Shih et al. (2016) examined the effect of using proprioceptive training in the form of game-based training with a Kinect sensor on postural stability in patients with Parkinson's disease. They found that proprioceptive training-induced improvements in both static and dynamic stability. Wang et al. (2018) studied the effect of using proprioceptive training in the form of game-based training on lower limb function and gait control in patients with spinocerebellar ataxia. They found that proprioceptive training caused an improvement in limb stability, limb-kinetic function, and gait-posture after 4 weeks.

\section{CONCLUSION}

Proprioceptive training can be an effective method for decreasing the incidence of muscle fatigue in patients with MNDs, particularly elderlies. This can be accomplished through the ability of proprioceptive training to normalize the firing rate of the $\alpha$-motoneurons and the amount of calcium released from calcium release channels, which has a major role in the occurrence of muscle fatigue. The normalization of $\alpha$-motoneurons and the amount of calcium released could be helpful to decrease the incidence of development of MNDs or to slow down the progression on presented MNDs in elderlies. Also, proprioceptive training decreases the occurrence of muscle fatigue by correcting the abnormal movement-compensations, which develop due to the biomechanical constraints imposed on patients with MNDs.

\section{AUTHOR CONTRIBUTIONS}

The author confirms being the sole contributor of this work and has approved it for publication.

Balsom, P. D., Gaitanos, G. C., Soderlund, K., and Ekblom, B. (1999). High-intensity exercise and muscle glycogen availability in humans. Acta Physiol. Scand. 165, 337-345. doi: 10.1046/j.1365-201x.1999. 00517.x

Bieñkiewicz, M. M. N., Rodger, M. W. M., Young, W. R., and Craig, C. M. (2013). Time to get a move on: overcoming bradykinetic movement in Parkinson's disease with artificial sensory guidance generated from biological motion. Behav. Brain Res. 253, 113-120. doi: 10.1016/j.bbr.2013.07.003

Black, C. D., O'connor, P. J., and McCully, K. K. (2005). Increased daily physical activity and fatigue symptoms in chronic fatigue syndrome. Dyn. Med. 4:3. doi: 10.1300/j092v04n04_02

Bongiovanni, L. G., and Hagbarth, K. E. (1990). Tonic vibration reflexes elicited during fatigue from maximal voluntary contractions in man. J. Physiol. 423, 1-14. doi: 10.1113/jphysiol.1990.sp018007 
Bowling, A. C., Schulz, J. B., Brown, R. H., and Beal, M. F. (1993). Superoxide dismutase activity, oxidative damage, and mitochondrial energy metabolism in familial and sporadic amyotrophic Lateral Sclerosis. J. Neurochem. 61, 2322-2325. doi: 10.1111/j.1471-4159.1993. tb07478.x

Boyas, S., and Guével, A. (2011). Neuromuscular fatigue in healthy muscle: underlying factors and adaptation mechanisms. Ann. Phys. Rehabil. Med. 54, 88-108. doi: 10.1016/j.rehab.2011.01.001

Brerro-Saby, C., Delliaux, S., Steinberg, J. G., and Jammes, Y. (2008). Fatigueinduced changes in tonic vibration response (TVR) in humans: relationships between electromyographic and biochemical events. Muscle Nerve 38, 1481-1489. doi: 10.1002/mus.21117

Broussalis, E., Grinzinger, S., Kunz, A. B., Killer-Oberpfalzer, M., Haschke-Becher, E., Hartung, H. P., et al. (2018). Late age onset of amyotrophic lateral sclerosis is often not considered in elderly people. Acta Neurol. Scand. 137, 329-334. doi: $10.1111 /$ ane. 12869

Cappello, V., and Francolini, M. (2017). Neuromuscular junction dismantling in amyotrophic lateral sclerosis. Int. J. Mol. Sci. 18:E2092.

Chen, S., and Hillman, D. E. (1999). Dying-back of Purkinje cell dendrites with synapse loss in aging rats. J. Neurocytol. 28, 187-196.

De Luca, C. J., and Kline, J. C. (2012). Influence of proprioceptive feedback on the firing rate and recruitment of motoneurons. J. Neural Eng. 9:016007. doi: 10.1088/1741-2560/9/1/016007

Dennett, A. M., Peiris, C. L., Shields, N., Prendergast, L. A., and Taylor, N. F. (2016). Moderate-intensity exercise reduces fatigue and improves mobility in cancer survivors: a systematic review and meta-regression. J. Physiother. 62, 68-82. doi: $10.1016 /$ j.jphys.2016.02.012

Dupuis, L., Gonzalez, J., Aguilar, D., and Oudart, H. (2005). Mitochondria in amyotrophic Lateral sclerosis: a trigger and a target. Neurodegener. Dis. 1, 245-254.

Dyck, P. J., Stevens, J. C., Mulder, D. W., and Espinosa, R. E. (1975). Frequency of nerve fiber degeneration of peripheral motor and sensory neurons in amyotrophic lateral sclerosis. Morphometry of deep and superficial peroneal nerves. Neurology 25, 781-785.

Edman, K. A. P., Radzyukevich, T., and Kronborg, B. (2002). Contractile properties of isolated muscle spindles of the frog. J. Physiol. 541, 905-916. doi: 10.1113/ jphysiol.2001.016220

Eisen, A., Schulzer, M., MacNeil, M., Pant, B., and Mak, E. (1993). Duration of amyotrophic lateral sclerosis is age dependent. Muscle Nerve 16, 27-32. doi: $10.1002 /$ mus. 880160107

Ellis, D. Z., Rabe, J., and Sweadner, K. J. (2003). Global loss of Na,K-ATPase and its nitric oxide-mediated regulation in a transgenic mouse model of amyotrophic lateral sclerosis. J. Neurosci. 23, 43-51. doi: 10.1523/jneurosci.23-01-00043. 2003

Eriksson, E. (2001). Can proprioception be trained? Knee Surg. Sport Traumatol. Arthrosc. 9, 127-127. doi: 10.1007/s001670100214

Fichna, J., Poole, D. P., Veldhuis, N., MacEachern, S. J., Saur, D., Zakrzewski, P. K., et al. (2015). Transient receptor potential vanilloid 4 inhibits mouse colonic motility by activating NO-dependent enteric neurotransmission. J. Mol. Med. 93, 1297-1309. doi: 10.1007/s00109-015-1336-5

Figlewicz, D. A., and Orrell, R. W. (2003). The genetics of motor neuron diseases. Amyotroph. Lateral Scler. Other Motor Neuron Disord. 4, 225-231. doi: 10.1080/ 14660820310011287

Fitts, R. H. (1994). Cellular mechanisms of muscle fatigue. Physiol. Rev. 74, 49-94. doi: 10.1152/physrev.1994.74.1.49

Forbes, R. B., Colville, S., and Swingler, R. J. (2004). The epidemiology of amyotrophic lateral sclerosis (ALS/MND) in people aged 80 or over. Age Ageing 33, 131-134. doi: 10.1093/ageing/afh013

Friedberg, F. (2002). Does graded activity increase activity? A case study of chronic fatigue syndrome. J. Behav. Ther. Exp. Psychiatry 33, 203-215. doi: 10.1016/ s0005-7916(02)00038-1

Fryer, M. W., Owen, V. J., Lamb, G. D., and Stephenson, D. G. (1995). Effects of creatine phosphate and $\mathrm{P}$ on $\mathrm{Ca}$ movements and tension development in rat skinned skeletal muscle fibres. J. Physiol. 482, 123-140. doi: 10.1113/jphysiol. 1995.sp020504

Gear, W. S. (2011). Effect of different levels of localized muscle fatigue on knee position sense. J. Sport Sci. Med. 10, 725-730.
Gibbons, C., Pagnini, F., Friede, T., and Ca, Y. (2018). Treatment of fatigue in amyotrophic lateral sclerosis/motor neuron disease. Cochane Database Syst. Rev. 1:CD011005.

Gibbons, C. J., Thornton, E. W., and Young, C. A. (2013). The patient experience of fatigue in motor neurone disease. Front. Psychol. 4:788. doi: 10.3389/fpsyg. 2013.00788

Green, H. J. (1997). Mechanisms of muscle fatigue in intense exercise. J. Sports. Sci. 15, 247-256. doi: 10.1080/026404197367254

Grunseich, C., and Fischbeck, K. H. (2015). Spinal and bulbar muscular atrophy. Neurol. Clin. 33, 847-854. doi: 10.1016/j.ncl.2015.07.002

Haidet-Phillips, A. M., Hester, M. E., Miranda, C. J., Meyer, K., Braun, L., Frakes, A., et al. (2011). Astrocytes from familial and sporadic ALS patients are toxic to motor neurons. Nat. Biotechnol. 29, 824-828. doi: 10.1038/nbt.1957

Hammad, M., Silva, A., Glass, J., Sladky, J. T., and Benatar, M. (2007). Clinical, electrophysiologic, and Pathologic evidence for sensory abnormalities in ALS. Neurology 69, 2236-2242. doi: 10.1212/01.wnl.0000286948.99150.16

Hausdorff, J. M., Lertratanakul, A., Cudkowicz, M. E., Peterson, A. L., Kaliton, D., Goldberger, A. L., et al. (2019). Dynamic markers of altered gait rhythm in amyotrophic lateral sclerosis. J. Appl. Physiol. 88, 2045-2053. doi: 10.1152/ jappl.2000.88.6.2045

Hepple, R. T. (2003). Sarcopenia-A critical perspective. Sci. Aging Knowl. Environ. 2003:e31.

Herndon, L. A., Schmeissner, P. J., Dudaronek, J. M., Brown, P. A., Listner, K. M., Sakano, Y., et al. (2002). Stochastic and genetic factors influence tissue-specific decline in ageing C. elegans. Nature 419, 808-814. doi: 10.1038/nature01135

Hospital, W. G., Road, C., and Hospital, S. G. (1993). Motor neuron disease: a disease of old age. Scott. Med. J. 38, 178-182. doi: 10.1177/003693309303800606

Hospod, V., Aimonetti, J.-M., Roll, J.-P., and Ribot-Ciscar, E. (2007). Changes in human muscle spindle sensitivity during a proprioceptive attention task. J. Neurosci. 27, 5172-5178. doi: 10.1523/jneurosci.0572-07.2007

Hutton, R. S., and Atwater, S. W. (1992). Acute and chronic adaptations of muscle proprioceptors in response to increased use. Sports Med. 14, 406-421. doi: 10.2165/00007256-199214060-00007

Hutton, R. S., and Nelson, D. L. (1986). Stretch sensitivity of golgi tendon organs in fatigued gastrocnemius muscle. Med. Sci. Sports Exerc. 18, 69-74.

Jang, Y. C., Sinha, M., Cerletti, M., Dall'Osso, C., and Wagers, A. J. (2011). Skeletal muscle stem cells: effects of aging and metabolism on muscle regenerative function. Cold Spring Harb. Symp. Quant. Biol. 76, 101-111. doi: 10.1101/sqb. 2011.76.010652

Jha, P., Ahmad, I., Khurana, S., Kamran, A., Verma, S., and Kumar, T. (2017). Proprioception: an evidence based narrative review. Res. Inves. Sport Med. 1, $1-5$.

Kang, H., and Lichtman, J. W. (2013). Motor axon regeneration and muscle reinnervation in young adult and aged animals. J. Neurosci. 33, 19480-19491. doi: 10.1523/JNEUROSCI.4067-13.2013

Kararizou, E., Manta, P., Kalfakis, N., and Vassilopoulos, D. (2005). Morphometric study of the human muscle spindle. Anal. Quant. Cytol. Histol. 27, 1-4.

Kaya, D. (ed.) (2016). "Exercise and Proprioception," in Proprioception: The Forgotten Sixth Sense, (Foster City, CA: OMICS Group).

Kent-Braun, J. A. (2009). Skeletal muscle fatigue in old age: whose advantage? Exerc. Sport Sci. Rev. 37, 3-9. doi: 10.1097/JES.0b013e318190ea2e

Klass, M., Benjamin, S., and Duchateau, J. (2007). Age-related fatigability of the ankle dorsiflexor muscles during concentric and eccentric contractions. Eur. J. Appl. Physiol. 100, 515-525. doi: 10.1007/s00421-006-0206-9

Knight, C. A., and Kamen, G. (2007). Modulation of motor unit firing rates during a complex sinusoidal force task in young and older adults. J. Appl. Physiol. 102, 122-129. doi: 10.1152/japplphysiol.00455.2006

Kuo, I. Y., and Ehrlich, B. E. (2015). Signaling in muscle contraction. Cold Spring Harb. Perspect. Biol. 7:a006023. doi: 10.1101/cshperspect.a006023

Kuppuswamy, A. (2017). The fatigue conundrum. Brain 140, 2240-2245. doi: 10.1093/brain/awx153

Kurtzke, J. F. (1991). Risk factors in amyotrophic lateral sclerosis. Adv. Neurol. 56, 245-270.

Li, L.-B., Lei, H., Arey, R. N., Li, P., Liu, J., Murphy, C. T., et al. (2016). The Neuronal kinesin UNC-104/KIF1A is a key regulator of synaptic aging and insulin signaling-regulated memory. Curr. Biol. 26, 605-615. doi: 10.1016/j.cub. 2015.12.068 
Light, A. R., Vierck, C. J., and Light, K. C. (2010). "Myalgia and fatigue: translation from mouse sensory neurons to fibromyalgia and chronic fatigue syndromes," in Translational Pain Research: From Mouse to Man, eds A. R. Light, and L. Kruger, (Boca Raton, FL: CRC Press/Taylor \& Francis), 1-23.

Liu, J., Zhang, B., Lei, H., Feng, Z., Liu, J., Hsu, A. L., et al. (2013). Functional aging in the nervous system contributes to age-dependent motor activity decline in C. elegans. Cell Metab. 18, 392-402. doi: 10.1016/j.cmet.2013.08.007

Liu, J.-X., Eriksson, P.-O., Thornell, L.-E., and Pedrosa-Domellöf, F. (2005). Fiber content and myosin heavy chain composition of muscle spindles in aged human biceps brachii. J. Histochem. Cytochem. 53, 445-454. doi: 10.1369/jhc.4a6257. 2005

MacIntosh, B. R., Holash, R. J., and Renaud, J.-M. (2012). Skeletal muscle fatigue - regulation of excitation-contraction coupling to avoid metabolic catastrophe. J. Cell. Sci. 125, 2105-2114. doi: 10.1242/jcs.093674

Marzetti, E., Lees, H. A., Manini, T. M., Buford, T. W., Aranda, J. M. Jr., Calvani, R., et al. (2012). Skeletal muscle apoptotic signaling predicts thigh muscle volume and gait speed in community-dwelling older persons: an exploratory study. PLoS One 7:e32829. doi: 10.1371/journal.pone.0032829

Masson, G. L., Przedborski, S., and Abbott, L. F. (2014). A computational model of motor neuron degeneration. Neurology 83, 975-988. doi: 10.1016/j.neuron. 2014.07.001

McCaskey, M. A., Schuster-Amft, C., Wirth, B., Suica, Z., and de Bruin, E. D. (2014). Effects of proprioceptive exercises on pain and function in chronic neck- and low back pain rehabilitation: a systematic literature review. $B M C$ Musculoskelet. Disord. 15:382. doi: 10.1186/1471-2474-15-382

McConell, G., Snow, R. J., Proietto, J., and Hargreaves, M. (1999). Muscle metabolism during prolonged exercise in humans: influence of carbohydrate availability. J. Appl. Physiol. 87, 1083-1086. doi: 10.1152/jappl.1999.87.3.1083

McElhiney, M. C., Rabkin, J. G., Gordon, P. H., Goetz, R., and Mitsumoto, H. (2009). Prevalence of fatigue and depression in ALS patients and change over time. J. Neurol. Neurosurg. Psychiatry 80, 1146-1149. doi: 10.1136/jnnp.2008. 163246

McKenzie, D., Bua, E., McKiernan, S., Cao, Z., Wanagat, J., and Aiken, J. M. (2002). Mitochondrial DNA deletion mutations: a causal role in sarcopenia. Eur. J. Biochem. 269, 2010-2015. doi: 10.1046/j.1432-1033.2002.02867.x

Mcneil, C. J., and Rice, C. L. (2007). Fatigability is increased with age during velocity-dependent contractions of the dorsiflexors. J. Gerontol. 62, 624-629. doi: 10.1093/gerona/62.6.624

Mentis, G. Z., Blivis, D., Liu, W., Drobac, E., Crowder, M. E., Kong, L., et al. (2011). Early functional impairment of sensory-motor connectivity in a mouse model of spinal muscular atrophy. Neuron 69, 453-467. doi: 10.1016/j.neuron.2010. 12.032

Mihevic, P. M. (1981). Sensory cues for perceived exertion: a review. Med. Sci. Sports Exerc. 13, 150-163.

Mochizuki, Y., Mizutani, T., Shimizu, T., and Kawata, A. (2011). Proportional neuronal loss between the primary motor and sensory cortex in amyotrophic lateral sclerosis. Neurosci. Lett. 503, 73-75. doi: 10.1016/j.neulet.2011.08.014

Mohamed, A., Jan, Y.-K., El Sayed, W. H., El Wanis, M. E. A., and Yamany, A. A. (2019). Dynamic scapular recognition exercise improves scapular upward rotation and shoulder pain and disability in patients with adhesive capsulitis: a randomized controlled trial. J. Man. Manip. Ther. [Epub ahead of print].

Morriss, R., Wearden, A., Mullis, R., Strickland, P. L., Pearson, D. J., Appleby, L., et al. (1996). A double-blind placebo controlled treatment trial of fluoxetine and graded exercise for chronic fatigue syndrome (CFS). Eur. Psychiatry 11:273s. doi: 10.1016/0924-9338(96)88824-9

Nelson, D. L., and Hutton, R. S. (1985). Dynamic and static stretch responses in muscle spindle receptors in fatigued muscle. Med Sci Sports Exerc 17, 445-450. doi: 10.1249/00005768-198508000-00007

Nijssen, J., Comley, L. H., and Hedlund, E. (2017). Motor neuron vulnerability and resistance in amyotrophic lateral sclerosis. Acta Neuropathol. 133, 863-885. doi: 10.1007/s00401-017-1708-8

Nogueira, D. V., Silva, S. B., de Abreu, L. C., Valenti, V. E., Fujimori, M., de Mello Monteiro, C. B., et al. (2012). Effect of the rest interval duration between contractions on muscle fatigue. Biomed. Eng. Online 11:89. doi: 10.1186/1475925X-11-89

Norris, F., Shepherd, R., Denys, E., Mukai, E., Elias, L., Holden, D., et al. (1993). Onset, natural history and outcome in idiopathic adult motor neuron disease. J. Neurol. Sci. 118, 48-55. doi: 10.1016/0022-510x(93)90245-t
Nunes, G. S., Bender, P. U., de Menezes, F. S., Yamashitafuji, I., Vargas, V. Z., and Wageck, B. (2016). Massage therapy decreases pain and perceived fatigue after long-distance Ironman triathlon: a randomised trial. J. Physiother. 62, 83-87. doi: 10.1016/j.jphys.2016.02.009

Nusbaum, N. J. (1999). Aging and sensory senescence. South Med. J. 92, 267-275. doi: 10.1097/00007611-199903000-00002

Oh, S. M., Bae, W. K., Choo, S. R., Kim, H. T., Kim, H. H., Lee, S. H., et al. (2016). Relationship between changes in fatigue and exercise by follow-up period. Korean J. Fam. Med. 37, 78-84. doi: 10.4082/kjfm.2016.37.2.78

Pan, C.-L., Peng, C.-Y., Chen, C.-H., and McIntire, S. (2011). Genetic analysis of age-dependent defects of the Caenorhabditis elegans touch receptor neurons. Proc. Natl. Acad. Sci. U.S.A. 108, 9274-9279. doi: 10.1073/pnas.101171 1108

Pandolf, K. B., Burse, R. L., and Goldman, R. F. (1975). Differentiated ratings of perceived exertion during physical conditioning of older individuals using legweight loading. Percept. Mot. Skills 40, 563-574. doi: 10.2466/pms.1975.40.2. 563

Philips, T., and Rothstein, J. D. (2014). Glial cells in amyotrophic lateral sclerosis. Exp. Neurol. 262PB, 111-120. doi: 10.1016/j.expneurol.2014.05.015

Potvin, J. R., and Fuglevand, A. J. (2017). A motor unit-based model of muscle fatigue. PLoS Comput. Biol. 13:e1005581. doi: 10.1371/journal.pcbi.1005581

Pugdahl, K., Fuglsang-Frederiksen, A., de Carvalho, M., Johnsen, B., Fawcett, P. R., Labarre-Vila, A., et al. (2007). Generalised sensory system abnormalities in amyotrophic lateral sclerosis: a European multicentre study. J. Neurol. Neurosurg. Psychiatry 78, 746-749. doi: 10.1136/jnnp.2006.098533

Quansah, E., and Karikari, T. K. (2015). Motor neuron diseases in subsaharan Africa: the need for more population-based studies. Biomed. Res. Int. 2015:298409. doi: 10.1155/2015/298409

Radovanović, S., Milićev, M., Perić, S., Basta, I., Kostić, V., and Stević, Z. (2014). Gait in amyotrophic lateral sclerosis: is gait pattern differently affected in spinal and bulbar onset of the disease during dual task walking? Amyotroph Lateral Scler. Frontotemporal Degener. 15, 488-493. doi: 10.3109/21678421. 2014.918148

Ribeiro, F., Mota, J., and Oliveira, J. (2007). Effect of exercise-induced fatigue on position sense of the knee in the elderly. Eur. J. Appl. Physiol. 99, 379-385. doi: 10.1007/s00421-006-0357-8

Ribeiro, F., and Oliveira, J. (2007). Aging effects on joint proprioception: the role of physical activity in proprioception preservation. Eur. Rev. Aging Phys. Act. 4, 71-76. doi: 10.1007/s11556-007-0026-x

Ribot-Ciscar, E., Rossi-Durand, C., and Roll, J. P. (2000). Increased muscle spindle sensitivity to movement during reinforcement manoeuvres in relaxed human subjects. J. Physiol. 523(Pt 1), 271-282. doi: 10.1111/j.1469-7793.2000.t01-100271.x

Riemann, B. L., and Lephart, S. M. (2002). The sensorimotor system, part II: the role of proprioception in motor control and functional joint stability. J. Athl. Train. 37, 80-84.

Rocha, M. C., Pousinha, P. A., Correia, A. M., Sebastião, A. M., and Ribeiro, J. A. (2013). Early changes of neuromuscular transmission in the SOD1(G93A) mice model of ALS start long before motor symptoms onset. PLoS One 8:e73846. doi: 10.1371/journal.pone.0073846

Rotto, D. M., and Kaufman, M. P. (1988). Effect of metabolic products of muscular contraction on discharge of group III and IV afferents. J. Appl. Physiol. 64, 2306-2313. doi: 10.1152/jappl.1988.64.6.2306

Sann, S. B., Crane, M. M., Lu, H., and Jin, Y. (2012). Rabx-5 regulates RAB-5 early endosomal compartments and synaptic vesicles in C. elegans. PLoS One 7:e37930. doi: 10.1371/journal.pone.0037930

Sharma, K. R., Kentbraun, J. A., Majumdar, S., Huang, Y., Mynhier, M., Weiner, M. W., et al. (1995). Physiology of fatigue in amyotrophic lateral sclerosis. Neurology 45, 733-740.

Shih, M. C., Wang, R. Y., Cheng, S. J., and Yang, Y. R. (2016). Effects of a balancebased exergaming intervention using the kinect sensor on posture stability in individuals with Parkinson's disease: a single-blinded randomized controlled trial. J. Neuroeng. Rehabil. 13:78.

Shumaker, R. G. (1980). The response of manual motor functioning in Parkinsonians to frontal EMG biofeedback and progressive relaxation. Biofeedback Self Regul. 5, 229-234. doi: 10.1007/bf00998598

Simmons, Z. (2013). Rehabilitation of Motor Neuron Disease, 1st Edn, Vol. 110. Amsterdam: Elsevier B.V. 
Soderberg, G. L., Minor, S. D., and Nelson, R. M. (1991). A comparison of motor unit behaviour in young and aged subjects. Age Ageing 20, 8-15. doi: 10.1093/ ageing/20.1.8

St Clair Gibson, A., Baden, D. A., Lambert, M. I., Lambert, E. V., Harley, Y. X., Hampson, D., et al. (2003). The conscious perception of the sensation of fatigue. Sports Med. 33, 1-10.

Stamford, B. A., and Noble, B. J. (1974). Metabolic cost and perception of effort during bicycle ergometer work performance. Med. Sci. Sports 6, 226-231.

Statland, J. M., Barohn, R. J., McVey, A. L., Katz, J., and Dimachkie, M. M. (2015). Patterns of weakness, classification of motor neuron disease \& clinical diagnosis of sporadic ALS. Neurol. Clin. 33, 735-748. doi: 10.1016/j.ncl.2015.07.006

Staud, R. (2012). Peripheral and central mechanisms of fatigue in inflammatory and non-inflammatory Rheumatic diseases. Curr. Rheumatol. Rep. 146, 539-548. doi: 10.1007/s11926-012-0277-z

Tank, E. M. H., Rodgers, K. E., and Kenyon, C. (2011). Spontaneous agerelated neurite branching in Caenorhabditis elegans. J. Neurosci. 31, 9279-9288. doi: 10.1523/JNEUROSCI.6606-10.2011

Terao, S., Miura, N., Osano, Y., Adachi, K., and Sobue, G. (2006). Clinical characteristics of elderly Japanese patients with amyotrophic lateral sclerosis; with special reference to the development of respiratory failure. Rinsho Shinkeigaku 46, 381-389.

Tibone, J. E., Fechter, J., and Kao, J. T. (2013). Evaluation of a proprioception pathway in patients with stable and unstable shoulders with somatosensory cortical evoked potentials. Committee. J. Shoulder Elbow Surg. 6, 440-443. doi: 10.1016/s1058-2746(97)70050-8

Tintignac, L. A., Brenner, H.-R., and Rüegg, M. A. (2015). Mechanisms regulating neuromuscular junction development and function and causes of muscle wasting. Physiol. Rev. 95, 809-852. doi: 10.1152/physrev.00033. 2014

Toth, M. L., Melentijevic, I., Shah, L., Bhatia, A., Lu, K., Talwar, A., et al. (2012). Neurite sprouting and synapse deterioration in the aging Caenorhabditis elegans nervous system. J. Neurosci. 32, 8778-8790. doi: 10.1523/JNEUROSCI.1494-11. 2012

Tysnes, O.-B., Vollset, S. E., Larsen, J. P., and Aarli, J. A. (1994). Prognostic factors and survival in amyotrophic lateral sclerosis. Neuroepidemiology 13, 226-235.

Valdez, G., Tapia, J. C., Kang, H., Clemenson, G. D. Jr., Gage, F. H., Lichtman, J. W., et al. (2010). Attenuation of age-related changes in mouse neuromuscular synapses by caloric restriction and exercise. Proc. Natl. Acad. Sci. 107, 14863-14868. doi: 10.1073/pnas. 1002220107

Vallbo, A. B., and Al-Falahe, N. A. (1990). Human muscle spindle response in a motor learning task. J. Physiol. 421, 553-568. doi: 10.1113/jphysiol.1990. sp017961

Vandoorne, T., De Bock, K., and Van Den Bosch, L. (2018). Energy metabolism in ALS: an underappreciated opportunity? Acta Neuropathol. 135, 489-509. doi: 10.1007/s00401-018-1835-x

Vaughan, S. K., Kemp, Z., Hatzipetros, T., Vieira, F., and Valdez, G. (2015). Degeneration of proprioceptive sensory nerve endings in mice harboring amyotrophic lateral sclerosis-causing mutations. J. Comp. Neurol. 523, 24772494. doi: $10.1002 /$ cne.23848

Vie, B., Gomez, N., Brerro-Saby, C., Weber, J. P., and Jammes, Y. (2013). Changes in stationary upright standing and proprioceptive reflex control of foot muscles after fatiguing static foot inversion. J. Biomech. 46, 1676-1682. doi: 10.1016/j jbiomech.2013.04.005

Vucic, S., Krishnan, A. V., and Kiernan, M. C. (2007). Fatigue and activity dependent changes in axonal excitability in amyotrophic lateral sclerosis. J. Neurol. Neurosurg. Psychiatry 78, 1202-1208. doi: 10.1136/jnnp.2006.112078

Wallman, K. E., Morton, A. R., Goodman, C., Grove, R., and Guilfoyle, A. M. (2004). Randomised controlled trial of graded exercise in chronic fatigue syndrome. Med. J. Aust. 180, 444-448.

Wan, J. J., Qin, Z., Wang, P. Y., Sun, Y., and Liu, X. (2017). Muscle fatigue: general understanding and treatment. Exp. Mol. Med. 49:e384. doi: 10.1038/emm.2017. 194

Wang, R. Y., Huang, F. Y., Soong, B. W., Huang, S. F., and Yang, Y. R. (2018). A randomized controlled pilot trial of game-based training in individuals with spinocerebellar ataxia type 3. Sci. Rep. 8:7816. doi: 10.1038/s41598-01826109-w

Watanabe, K., Holobar, A., Kouzaki, M., Ogawa, M., Akima, H., and Moritani, T. (2016). Age-related changes in motor unit firing pattern of vastus lateralis muscle during low-moderate contraction. Age 38:48. doi: 10.1007/s11357-0169915-0

Weisleder, N., Brotto, M., Komazaki, S., Pan, Z., Zhao, X., Nosek, T., et al. (2006). Muscle aging is associated with compromised Ca2+ spark signaling and segregated intracellular Ca2+ release. J Cell Biol 174, 639-645. doi: 10.1083/jcb. 200604166

White, L. E., and Hall, W. C. (2018). "Lower Motor Neuron Circuits and Motor Control," in Neuroscience, 6th Edn, eds D. Purves, G. J. Augustine, and D. Fitzpatrick, (New York, NY: Sinauer Associates), 357-380.

Witiuk, K., Fernandez-Ruiz, J., McKee, R., Alahyane, N., Coe, B. C., Melanson, M., et al. (2014). Cognitive deterioration and functional compensation in ALS measured with fMRI using an inhibitory task. J. Neurosci. 34, 14260-14271. doi: 10.1523/JNEUROSCI.1111-14.2014

Wu, Y., and Ng, S. C. (2010). A PDF-Based Classification of Gait Cadence Patterns in Patients with Amyotrophic Lateral Sclerosis. Annu. Int. Conf. IEEE Eng. Med. Biol. 2010, 1304-1307. doi: 10.1109/IEMBS.2010.5626398

Wu, Y., and Shi, L. (2011). Analysis of altered gait cycle duration in amyotrophic lateral sclerosis based on nonparametric probability density function estimation. Med. Eng. Phys. 33, 347-355. doi: 10.1016/j.medengphy. 2010.10.023

Yong, M.-S., and Lee, Y.-S. (2017). Effect of ankle proprioceptive exercise on static and dynamic balance in normal adults. J. Phys. Ther. Sci. 29, 242-244. doi: $10.1589 /$ jpts.29.242

Conflict of Interest: The author declares that the research was conducted in the absence of any commercial or financial relationships that could be construed as a potential conflict of interest.

Copyright (c) 2019 Mohamed. This is an open-access article distributed under the terms of the Creative Commons Attribution License (CC BY). The use, distribution or reproduction in other forums is permitted, provided the original author(s) and the copyright owner(s) are credited and that the original publication in this journal is cited, in accordance with accepted academic practice. No use, distribution or reproduction is permitted which does not comply with these terms. 